\title{
An approach for decentralized mode estimation based on the Random Decrement method
}

\author{
A. Friedmann*, D. Mayer and M. Kauba \\ Fraunhofer Institute for Structural Durability and System Reliability, Bartningstr. 47, 64289 Darmstadt, Germany
}

\begin{abstract}
Structural health monitoring methods based on modal properties have been proven to be well suited for infrastructure objects, e.g. bridges, buildings or wind turbines. The considerable size of these structures leads to long distances between the sensors and the signal processing units and a large number of sensors. To save cabling effort and lower the amount of data which has to be transmitted, the structural analysis may be decentralized with a network of smart sensors.

In this paper, a strategy for decentralized signal analysis with the Random Decrement method is discussed. This method allows for the estimation of auto- and cross-correlation functions simply from triggered averaging of time histories. These serve as a basis for the modal decomposition by operational modal analysis methods. While the Random Decrement signatures can be processed in a decentralized way on the small microcontrollers commonly used, e.g. in wireless sensing applications, the deeper analysis of resonance frequencies and mode shapes is done by a central unit.

After a short introduction into the theory of the Random Decrement method, its application in decentralized data acquisition is illustrated with a numerical example. Afterwards, a simple experimental structure exposed to actual wind loads is instrumented and the decentralized signal processing strategy is tested with the acquired data. A first algorithm for the modal decomposition of the estimated correlation functions is implemented and tested.
\end{abstract}

Keywords: Modal analysis, modal testing, structural health monitoring

\section{Introduction}

Structural health monitoring (SHM) is known as the process of in-service damage detection for aerospace, civil, and mechanical engineering infrastructure and is a key element of strategies for condition based maintenance and damage prognosis [1]. Especially for the monitoring of large infrastructure objects like buildings, bridges or wind turbines, vibration based damage detection methods have been proven as well suited. Most of the methods incorporate the identification of structural dynamic characteristics from vibration measurements and have been tested in the laboratory as well as with actual structures for many years [2].

Recently more attention has been drawn to the transfer of structural health monitoring methods to practical applications, including issues of system integration [3]. Highly integrated MEMS acceleration sensors as standard off-the-shelf products [4] together with the development of miniaturized signal processing platforms for the setup of smart sensor networks offer interesting possibilities for the realization of a monitoring system including a high number of sensors distributed widespread over a large mechanical structure [5]. This approach should reduce the efforts of cabling considerably, even when using wire-connected sensor nodes (Fig. 1). However, the use of communication channels, especially wireless, raises challenges like limited bandwidth for the transmission of data, synchronization and reliable data transport [6]. Thus, it is desirable to use the nodes of the sensor network not

\footnotetext{
*Corresponding author. E-mail: andreas.friedmann@1bf.fraunhofer.de.
} 

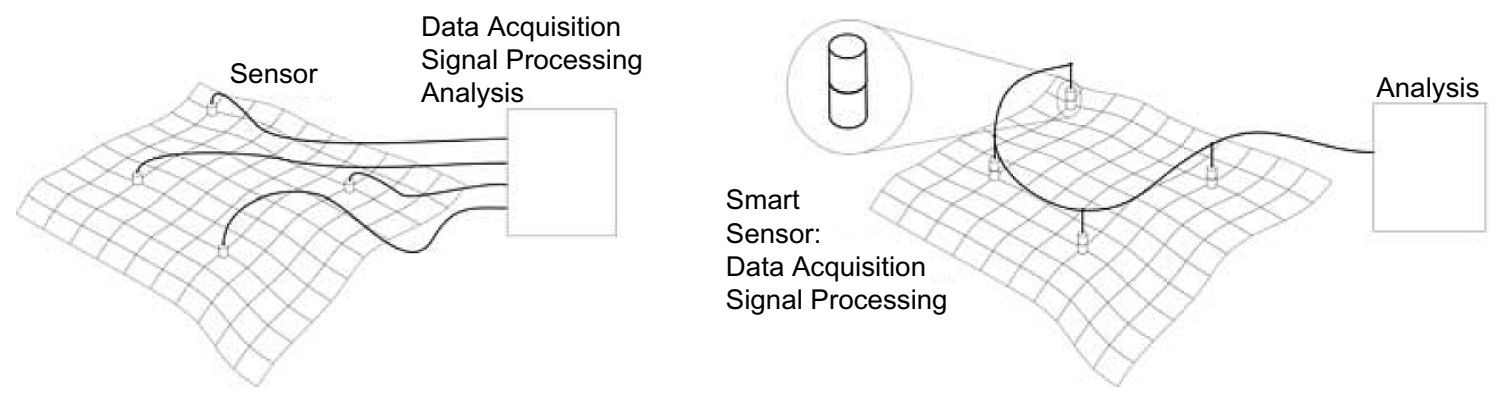

Fig. 1. SHM system with centralized acquisition and processing unit versus system with smart sensors.

only for data acquisition and transmission, but also for the local pre-processing of the data in order to compress the amount of transmitted data. For instance, basic calculations like spectral estimation of the acquired data sequences can be implemented [7]. The microcontrollers usually applied in wireless sensor platforms are mostly not capable to perform extensive calculations. Therefore, the algorithms for local processing should possess a low computational effort.

In this paper, the Random Decrement (RD) method is evaluated with respect to distributed signal processing on sensor nodes, which is a simple, yet effective method for the estimation of correlation functions [8,9]. It was originally invented for the damage detection of aerospace structures under random loading, but can also successfully be applied as a component of a structural parameter identification, e.g. in operational modal analysis [10,11]. Having estimated averaged correlation functions by means of the smart sensors, these functions are transferred to a central processing unit. There, the algorithms of Frequency Domain Decomposition (FDD) are applied to estimate eigenfrequencies of the system under test.

\section{Description of the Random Decrement technique}

Most of the structures which are interesting for SHM cannot be excited for structural analysis, either because they are too large (e.g. infrastructure objects) or because it is impractical to apply a vibration exciter during operation. Thus only the output signals, i.e. the vibrations excited by operational loads are used in order to estimate auto- and cross-correlation functions. The concept of the RD technique is a simple method to estimate the auto-correlation function of a system under random input loads by averaging time series which are taken when a given trigger condition is fulfilled. The method can be explained descriptively in the following way.

At each time instant, the response of the system is composed of three parts: The response to an initial displacement, the response to an initial velocity and the response to the random input loads during the time span between the initial state and the regarded time instant [10]. By averaging many of those time series, the random part will disappear, while the result is a scaled estimation of the auto-correlation which may be interpreted as the free decay of the system in case of a white noise excitation.

The concept may be extended to the estimation of cross-correlation functions between two system outputs. This is simply achieved by generating averaged time series by defining a trigger condition for the first output $x$, but synchronously taking time series of other outputs. If a simple level crossing of the signal $x$ is assumed, the mathematical expression of the RD technique reads [10]:

$$
D_{X X}(\tau)=\frac{1}{N} \sum_{n=1}^{N} x\left(t_{n}+\tau\right)\left|x\left(t_{n}\right)=a \quad D_{Y X}(\tau)=\frac{1}{N} \sum_{n=1}^{N} y\left(t_{n}+\tau\right)\right| x\left(t_{n}\right)=a
$$

The method should be illustrated with a simple example, a time history of a signal and the definition of a level crossing trigger (Fig. 2). In the lower part of the plot, three examples for recorded and averaged time series are shown.

This method could be decentralized using the network architecture shown in Fig. 3. It is important to mention that during data acquisition only trigger signals have to be shared via the network. The transfer of spectral data is required only after every sensor node has finished its averaging process. At this point the speed of the data transfer to the central unit is of minor importance to the general performance of the system. 



Fig. 2. Example for the calculation of a RD signature with a simple level crossing trigger condition. Upper part: time history, lower part: Time sequences (solid) and averaged RD signature (dashed).

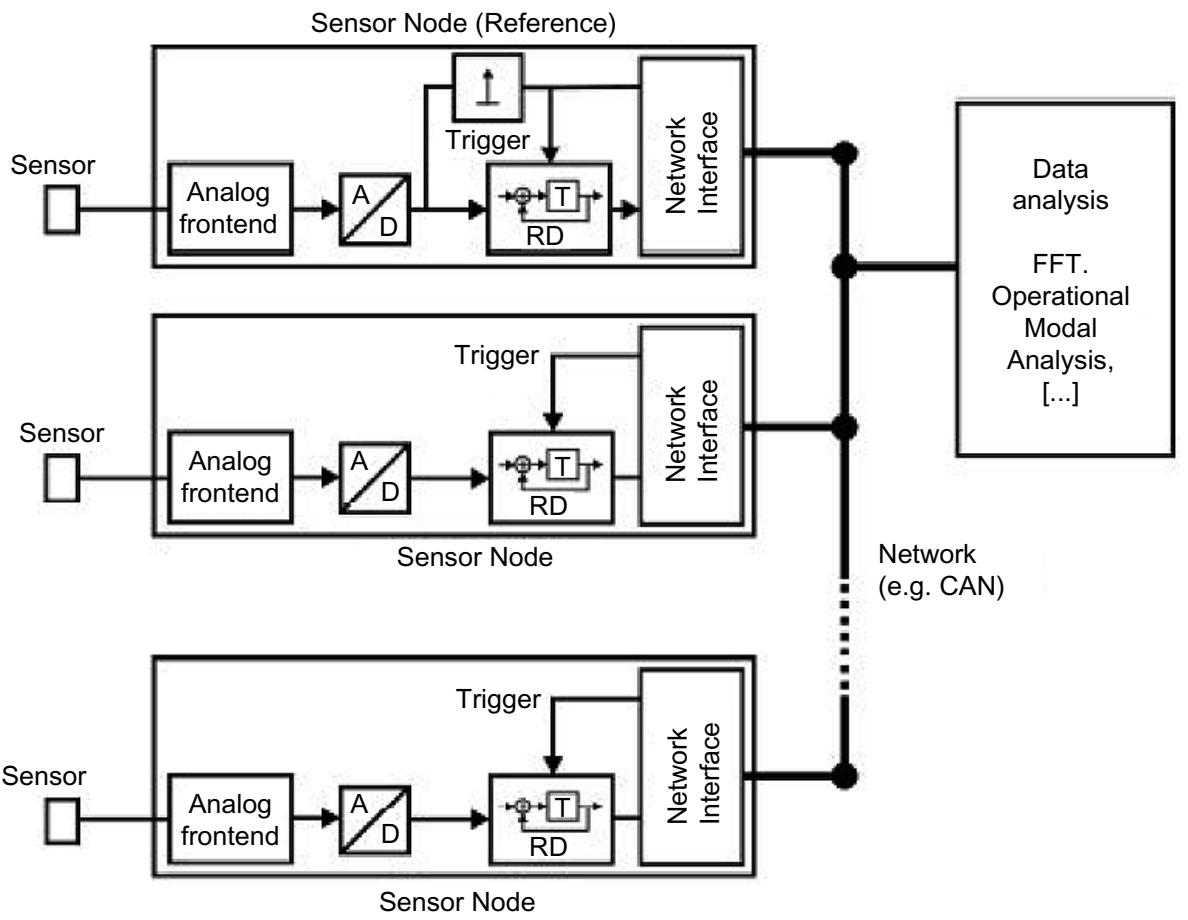

Fig. 3. System layout for decentralized RD estimation and subsequent data analysis. 


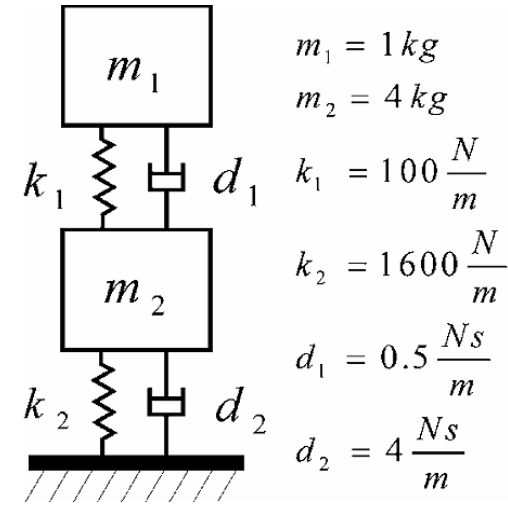

Fig. 4. Two degree-of-freedom system for numerical investigations.

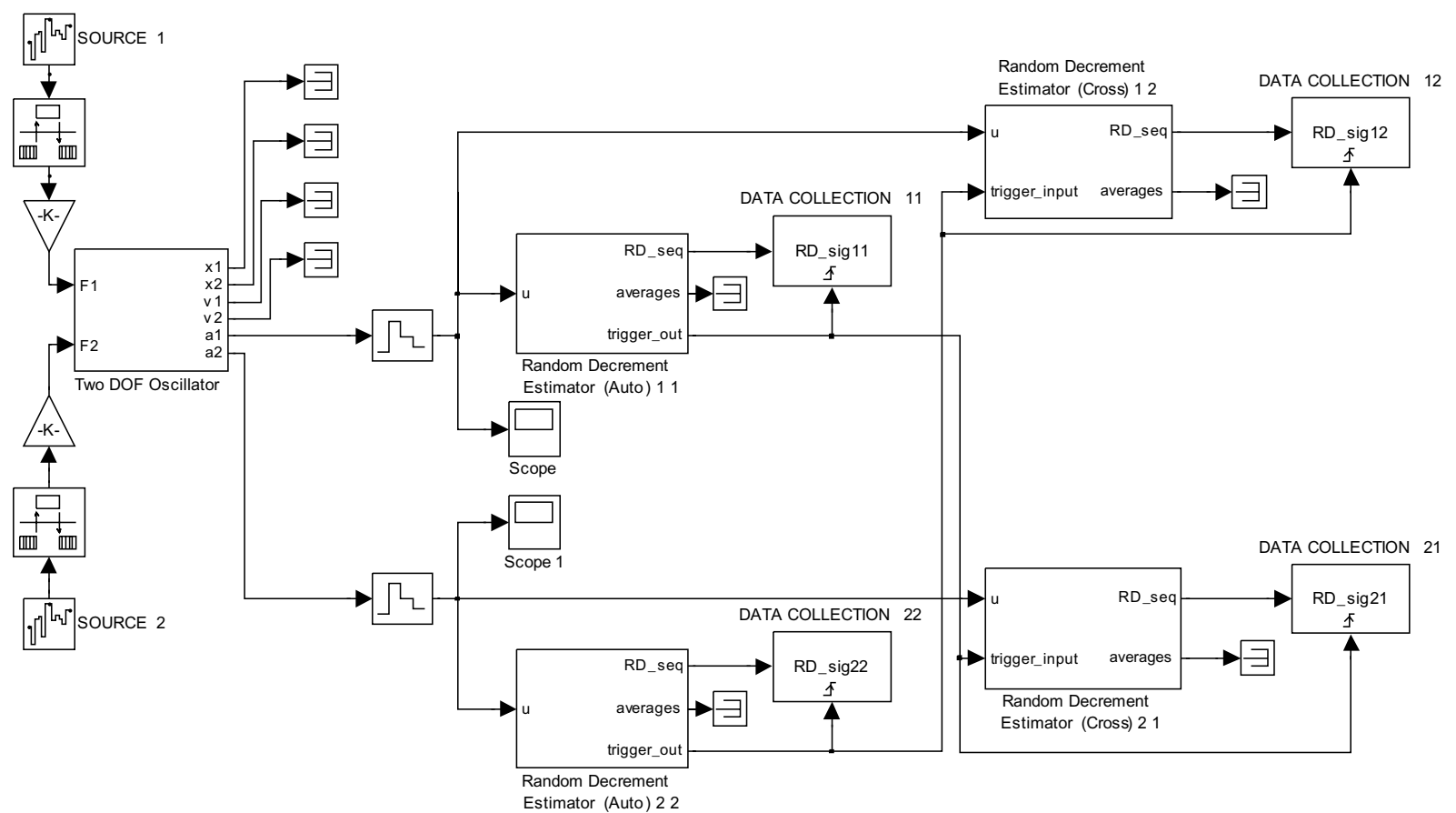

Fig. 5. Time domain system simulation of a mechanical system and the RD estimation.

\section{Application to a system simulation}

The properties of the RD method should be studied by a system simulation in order to obtain hints for potential implementation with a decentralized sensor network. Therefore, a mechanical structure is represented by a system with two oscillatory degrees of freedom (Fig. 4). The eigenfrequencies of this system calculated by analyzing the system matrices are $1.53 \mathrm{~Hz}$ and $3.30 \mathrm{~Hz}$.

Band-limited Gaussian white noise serves as force excitation, while accelerations are used as outputs, since accelerometers are the most common sensors in structural analysis of flexible structures. The simulation is set up in time domain using Simulink including the mechanical system as a time-continuous model and the RD estimators for auto- and cross-correlation as discrete-time sub-models at a sampling interval of $0.005 \mathrm{~s}$ which is equal to a sampling frequency of $200 \mathrm{~Hz}$ (Fig. 5). Both outputs are used as the references, thus the trigger condition is checked for both signals. In case of a trigger event, the auto RD estimator block sends a trigger signal to the connected cross RD 



Fig. 6. RD signatures of the numerical model.

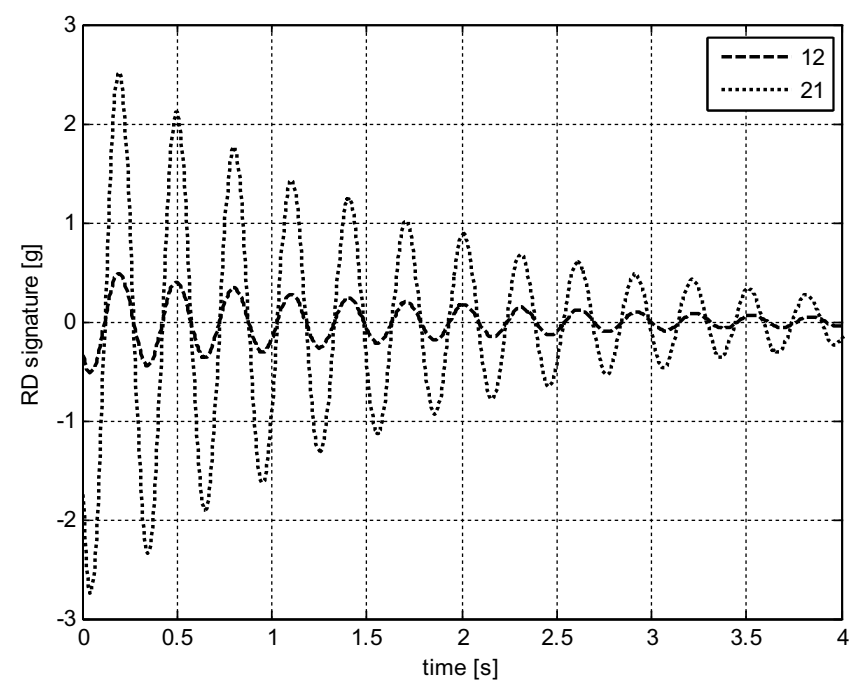

Fig. 7. Comparison of RD signatures 12 and 21 of numerical model.

estimator block of the second system output. All RD estimator blocks perform the averaging process of the triggered time sequences decentralized and provide the results at their outputs. The number of averages for the signatures shown below is greater than 1000 .

The RD signatures are estimated with a length of 2048 elements and are shown in Fig. 6. Estimating the signatures with all possible combinations of measurement channels and trigger channels leads to a square matrix of RD signatures of the size of $\mathrm{N} \times \mathrm{N}$ where $\mathrm{N}$ is the number of sensors in use (here: $2 \times 2$ ). The difference of amplitude of the RD signatures 12 and 21 (Fig. 7) can be explained by the use of different trigger levels. A comparison is only possible if the signatures are normalized with respect to the trigger values because the trigger level directly determines the first value of the signatures. In the presented case, this would lead to fitting curves. In further analyses, this problem can be dealt with in different ways. It is either possible to use the same trigger levels in all channels, or to normalize the estimated signatures with respect to the used trigger level. In the presented work, this was not necessary, because only eigenfrequencies are estimated, but such normalization is needed for proper mode shape estimation. The additional process might be implemented in the sensor nodes, so the need for normalization does 

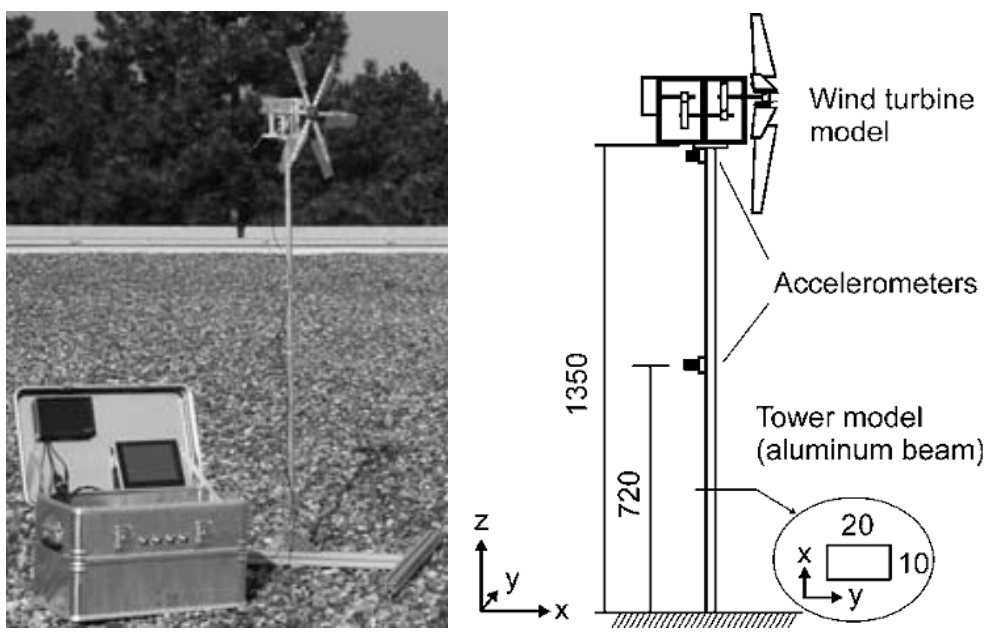

Fig. 8. Mechanical system for experimental investigations.
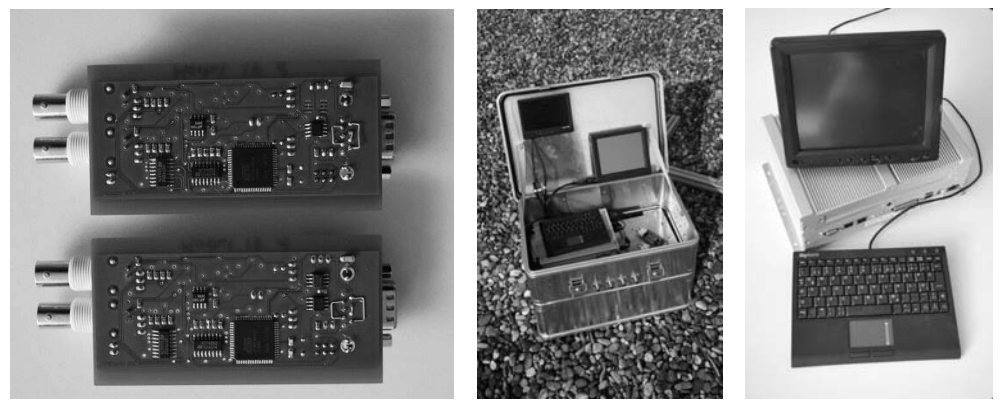

Fig. 9. Realisation of the sensor nodes and the central processing unit.

not effect the idea of decentralization.

\section{Experimental set-up}

To test the performance of the decentralized signal analysis in reality, a structure is chosen which is exposed to actual environmental excitations by wind loads. A small model of a wind turbine (weight approx. $0.5 \mathrm{~kg}$ ) is mounted on top of an aluminum beam, which serves as a model for the tower (Fig. 8). Although quite simple and small, the wind turbine model possesses a gearbox with several stages which may serve as a potential noise source during operation. The cross-section and length of the beam are chosen properly, so that the resonance frequencies of the assembled system are in a range similar to those of a full scale structure. Since the cross-section of the beam is rectangular, the bending eigenmodes in $\mathrm{x}$ and $\mathrm{y}$ direction should possess different eigenfrequencies in order to alleviate the structural analysis. For the first tests, the beam is instrumented with two MEMS-type accelerometers, which have been realized on basis of a commercial integrated circuit ADXL322 with a sensitivity of $0.42 \mathrm{~V} / \mathrm{g}$ and a measurement range of $\pm 2 \mathrm{~g}$ [4].

One sensor is mounted nearly at the top of the beam (sensor 1) and the other at half length (sensor 2) (Fig. 8). Since the accelerometers possess two axes each, measurements of the tower vibrations in both bending directions are possible. For the first test presented here, only the x-axis is used.

As a reference for the measurements under operational conditions a frequency response measurement is conducted in the laboratory. The tower is excited with an impulse hammer at the position of sensor 2, while the response is acquired with an attached accelerometer at both sensor positions. In the regarded $\mathrm{x}$-direction, the first two bending modes occur at $4.5 \mathrm{~Hz}$ and $34.0 \mathrm{~Hz}$. 

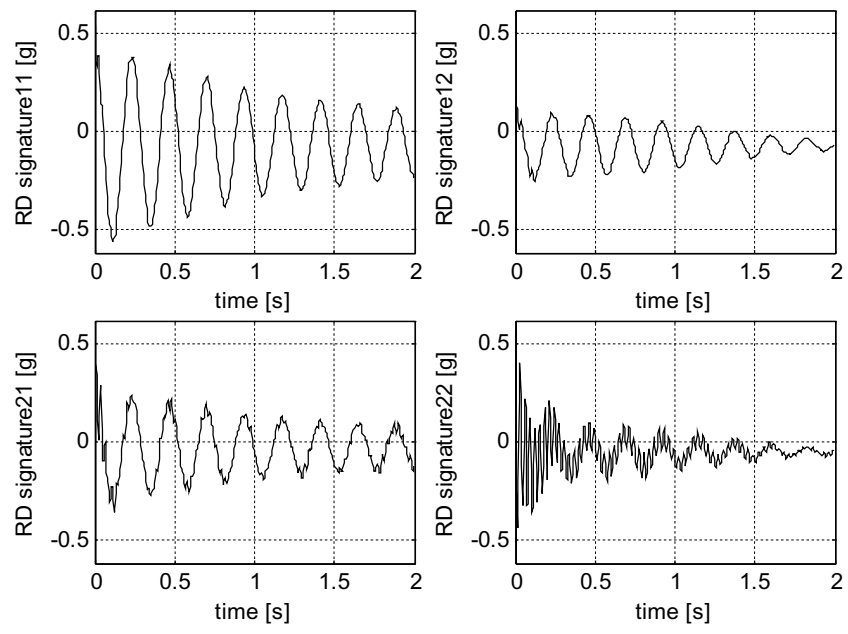

Fig. 10. RD signatures of experimental setup.

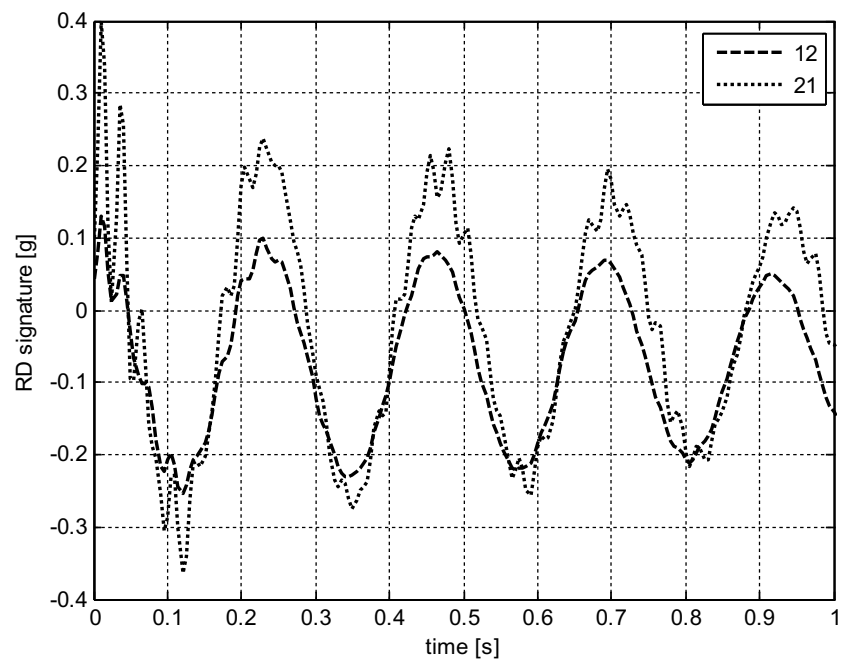

Fig. 11. Comparison of RD signatures 12 and 21 of experimental set-up.

To evaluate the basic functionality of the RD technique, the signal processing algorithm is implemented on a microcontroller for the sensor nodes and an embedded PC for the central processing unit (Fig. 9), both using automatic code generation from Simulink (a deeper insight in the architecture of the acquisition system and its extensions is given in [13]). Since both sensor signals are processed on one hardware platform this is considered as a step for functional prototyping of the algorithms and the system layout in general. A sampling rate of $200 \mathrm{~Hz}$ was chosen, high enough to acquire vibrations related to the first few bending modes. The RD signatures' length was set to 400 elements; the number of averages for the shown signatures is 1024 .

The estimated RD signatures are show in Fig. 10; like before, a comparison of the cross RD signatures is shown in Fig. 11. It can be seen, that the quality of the experimental results is comparable to those results gathered numerically.

\section{Modal decomposition of estimated correlation functions}

The idea described before was to estimate correlation functions by the smart sensors; transfer them to a central unit and perform the modal analysis on this unit. In the application described here, this central unit is a common 
Table 1

Measured / calculated and estimated eigenfrequencies

\begin{tabular}{lccc}
\hline Model under test & Mode & \multicolumn{2}{c}{ Eigenfrequency [Hz] } \\
\cline { 3 - 4 } & & Calculated / measured & Estimated by FDD \\
\hline numerical model & 1. & 1.53 & 1.56 \\
& 2. & 3.30 & 3.32 \\
experimental setup & 1. & 4.50 & 4.50 \\
& 2. & 34.0 & 32.0 \\
\hline
\end{tabular}
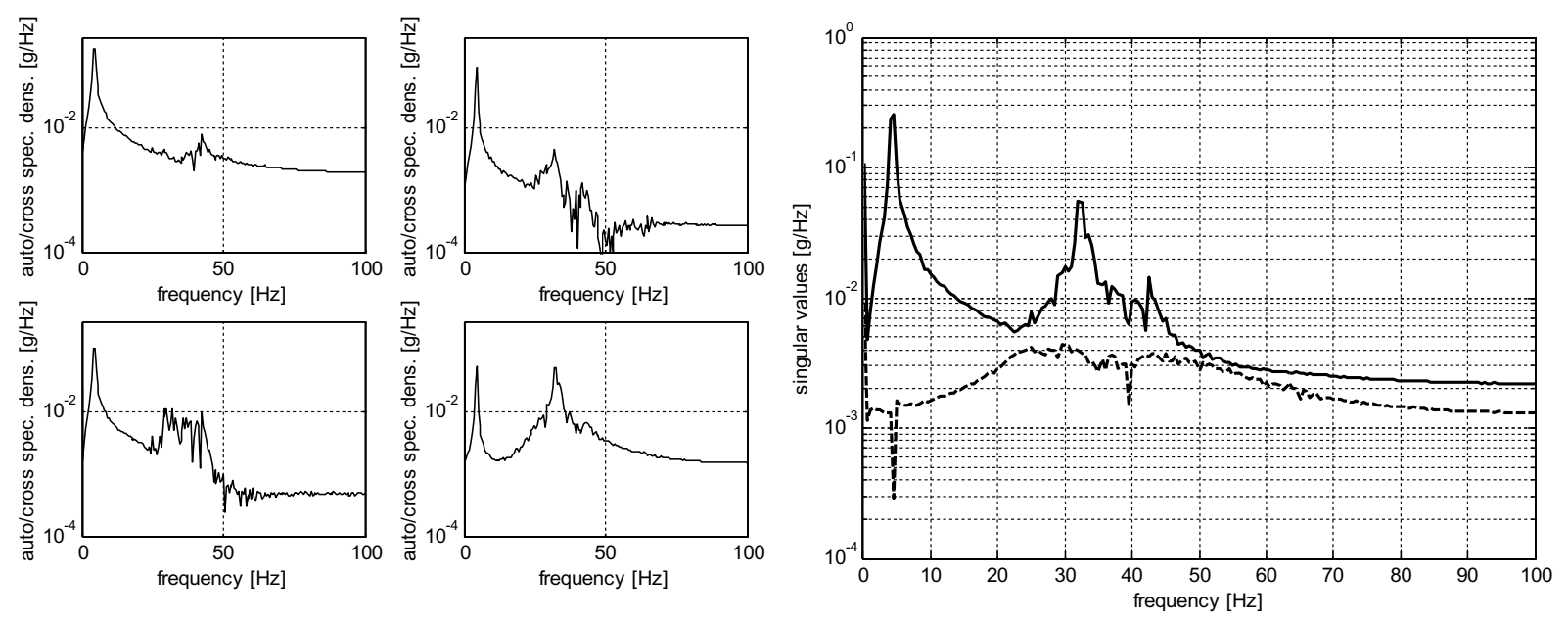

Fig. 12. Auto and cross spectral densities and singular values of experimental setup.

desktop computer where the matrices of the correlation function are evaluated by a matlab routine. This routine includes reading the correlation matrices from different file types (*.mat for the numerical model, *.xml for the experimental setup), calculating the spectral densities matrix by means of a single block DFT [14] without using time windowing and evaluating this matrix by means of frequency domain operational modal analysis, namely, the FDD-algorithm. This algorithm described first by Brincker et al. [10] is based on a Singular Value Decomposition of every frequency line of the spectral densities matrix. This process leads to a diagonal matrix holding the spectra of the so-called singular values in decreasing order. The peak values of the first singular value then are interpreted as indicators for the frequency of the systems' eigenmodes.

In Fig. 12 and in Fig. 13, the matrices of the spectral densities and the spectra of the singular values are shown for the numerical model and the experimental set-up, respectively. In both cases, it can be seen that the values of the eigenfrequencies estimated by means of the singular value are very close to the frequencies measured directly (see Table 1). Furthermore, again the reciprocity of the matrix can be seen.

\section{Conclusions}

In this paper, a strategy for the acquisition of vibration data based on a smart sensor network is presented. This acquisition is based on the decentralized application of the RD technique. This technique is implemented with very simple computations, compared to FFT based estimation methods. The averaging process in general requires just adding up a series of time blocks and a final division of the sum by the number of blocks summed up. Furthermore, both of the RD estimators include a very effective data compression, since the averaged RD signatures may be constructed from very long time series.

Afterwards the use of the frequency domain operational modal analysis acquired for and performed on a central unit is explained. The use of both, the decentralized data acquisition and the centralized data analysis, is shown on a numerical model and an experimental set-up. It can be seen that in both cases the system works well with regard to 

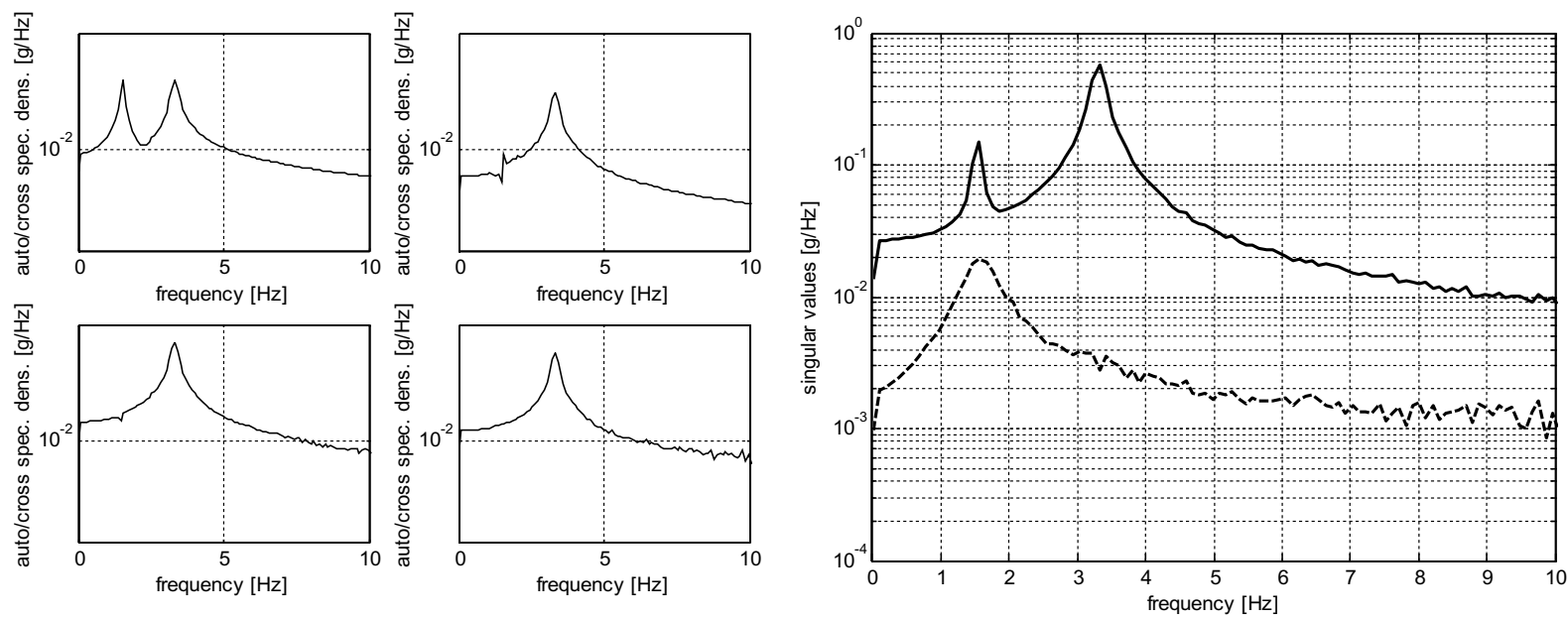

Fig. 13. Auto and cross spectral densities and singular values of the numerical model.

estimating the eigenfrequencies of the respective system. Even if such an estimation of the eigenfrequencies might be done easier without taking in account the phase behaviour [15], this information is needed for the further work, namely estimating the eigenmodes of the system under test. Those and the information about the modal damping, which should be obtained as well, will be important for the application of the data acquisition system in Structural Health Monitoring. Therefore, it is needed that the modal extraction can be automated to perform modal analysis without user influence, so that the developement of the modal parameters can be measured over a certain period of time.

\section{Acknowledgement}

The authors wish to thank Mr. Michael Koch, who conducted the experimental work.

\section{References}

[1] C.R. Farrar et al., Damage Prognosis: Current Status and Future Needs, Los Alamos National Laboratory Report, LA-14051-MS, 2003.

[2] C.R. Farrar and S.W. Doebling, An Overview of Modal-Based Damage Identification Methods, EUROMECH 365 International Workshop: DAMAS 97, 1997.

[3] H. van der Auweraer and B. Peeters, Discriminating Physical Poles From Mathematical Poles In High Order Systems: Use And Automation Of The Stabilization Diagram, Proceedings of the IEEE Instrumentation and Measurement Technology Conference, 2004.

[4] N.N.: Analog Devices - Small and Thin \pm 2 g Accelerometer ADXL322, data sheet.

[5] B.F. Spencer, M. Ruiz-Sandoval and N. Kurata, Smart sensing technology: opportunities and challenges, Structural Control and Health Monitoring 11(4) (2004), 349-368.

[6] N. Xu et al., A Wireless Sensor Network For Structural Monitoring, SenSys'04, Baltimore, Maryland, USA, 2004.

[7] J. Lynch, Y. Wang, J.L. Kenneth, J.H. Yi and C.-B. Yun, Performance Monitoring of the Geumdang Bridge using a Dense Network of High-Resolution Wireless Sensors, Smart Materials and Structures, 2006, pp. 1561-1575.

[8] H. Cole, On-Line Failure Detection and Damping Measurement of Aerospace Structures by Random Decrement Signatures, Report NASA CR-2205, 1973.

[9] R. Brincker, S. Krenk and J.L. Jensen, Estimation Of Correlation Functions By The Random Decrement, Proceedings of The Florence Modal Anaylsis Conference, 1991, pp. 783-788.

[10] J. Rodrigues and R. Brincker, Application of the Random Decrement Technique in Operational Modal Analysis, Proceedings of the 1st International Operational Modal Analysis Conference (IOMAC), Copenhagen, Denmark, 2005.

[11] J.C. Asmussen, Modal Analysis Based on the Random Decrement Technique - Application to Civil Engineering Structures, PhD-Thesis, 1998, University of Aalborg, Aalborg, Denmark.

[12] R. Brincker, L. Zhang and P. Andersen, Modal Identification form Ambient Response using Frequency Domain Decomposition, Proceedings of the 18th IMAC, 2000.

[13] D. Mayer, M. Kauba and A. Friedmann, An Approach for Structural Health Monitoring with Smart Sensor Networks Based on the Random Decrement Method, Proc. of ECCOMAS Thematic Conference on Smart Structures and Materials (SMART), Porto, Portugal, 2009. 
[14] K.G. McConnell, Vibration Testing - Theory and Practice, New York: John Wiley \& Sons, Inc. 1995.

[15] G. Feltrin, Environmental Effects on Eigenfrequencies of a RC Highway Bridge, European meeting on intelligent structures, Ischia, Italy, September 22-28, 2001. 

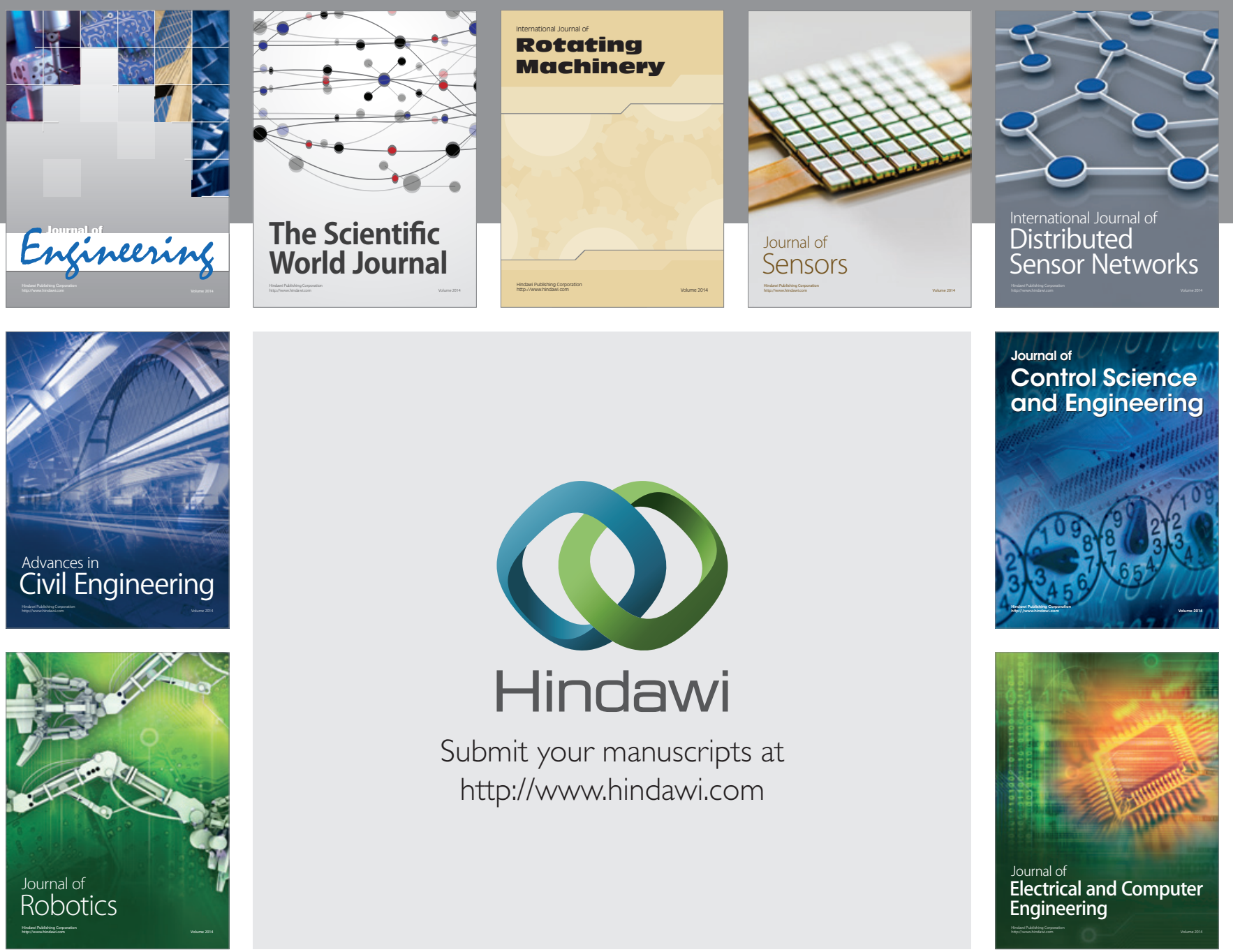

Submit your manuscripts at

http://www.hindawi.com
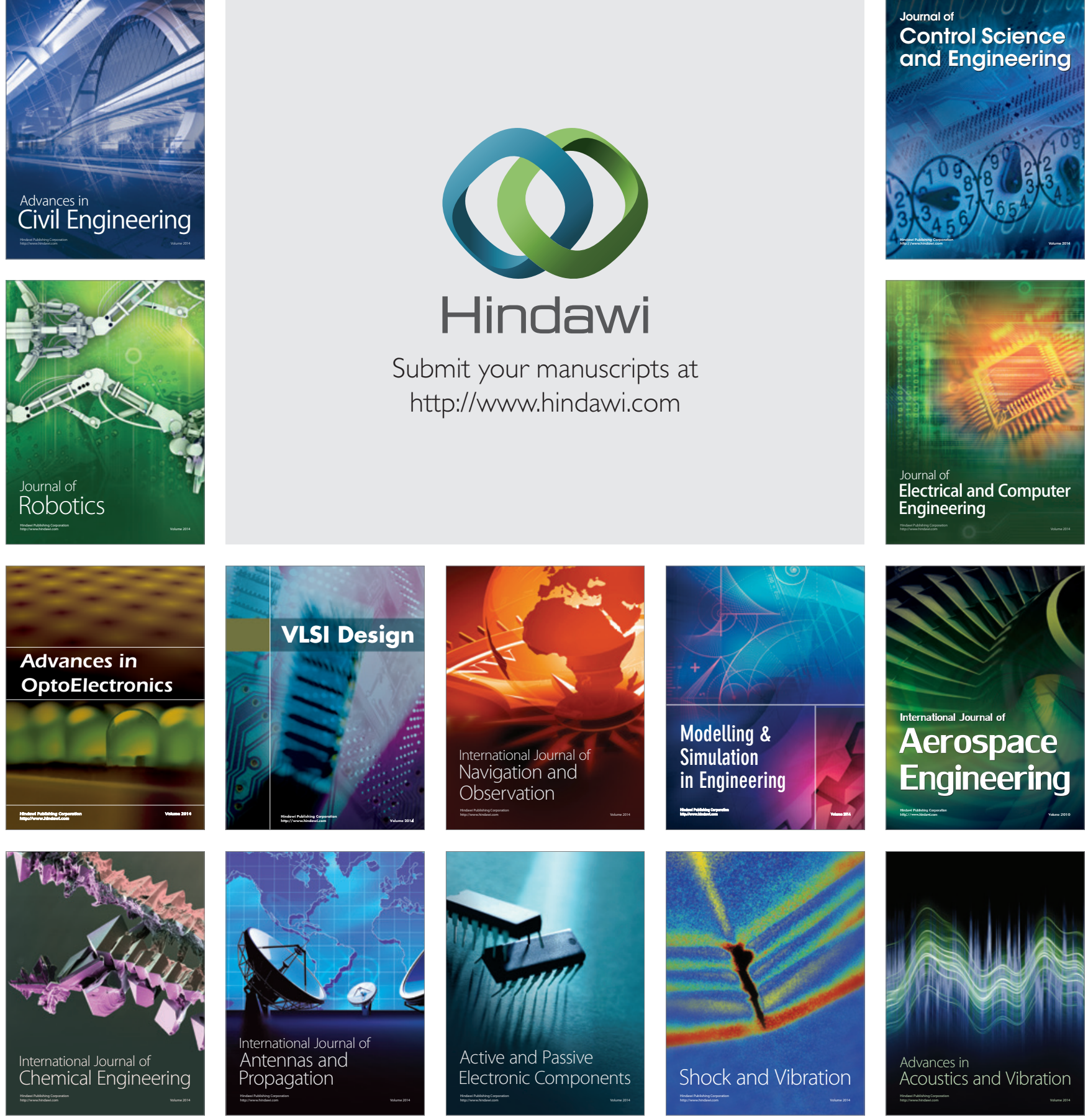